PROCEEDINGS OF THE

AMERICAN MATHEMATICAL SOCIETY

Volume 138, Number 10, October 2010, Pages 3591-3601

S 0002-9939(10)10361-X

Article electronically published on April 27, 2010

\title{
MATHER MEASURES SELECTED BY AN APPROXIMATION SCHEME
}

\author{
DIOGO GOMES, RENATO ITURRIAGA, HÉCTOR SÁNCHEZ-MORGADO, \\ AND YIFENG YU
}

(Communicated by Yingfei Yi)

\begin{abstract}
In this note, we will identify Mather measures selected by Evans's variational approach in 1-d. Motivated by the low dimension case, we conjecture that Evans's approximation scheme might catch the whole Mather set in all dimensions. We also discuss the connection with another approximation scheme in the works of Anantharaman, Evans and Gomes.
\end{abstract}

\section{INTRODUCTION}

Let $\mathbb{T}^{n}$ denote the $n$-dimensional flat torus. Suppose that $H \in C^{\infty}\left(\mathbb{R}^{n} \times \mathbb{T}^{n}\right)$ satisfies the following standard assumptions:

(i) (Superlinearity)

$$
\lim _{|p| \rightarrow+\infty} \frac{H(p, x)}{|p|}=+\infty \quad \text { uniformly in } \mathbb{T}^{n} .
$$

(ii) (Strict convexity) The Hessian matrix is such that

$$
\left[H_{p_{i} p_{j}}(p, x)\right]>0 \quad \text { for each }(p, x) \in \mathbb{R}^{n} \times \mathbb{T}^{n} .
$$

The famous Kolmogorov-Arnold-Moser (KAM) theory says that if a Hamiltonian system is near integrable, most invariant tori will be preserved. However, the invariant tori will be destroyed when the perturbation becomes large. According to the Aubry-Mather theory, the most important remnant is the Mather set. We write $\mathcal{I}$ for the set of Euler-Lagrangian flow invariant probability Borel measures on $\mathbb{R}^{n} \times \mathbb{T}^{n}$. Let

$$
-c_{H}=\inf _{\nu \in \mathcal{I}} \int_{\mathbb{R}^{n} \times \mathbb{T}^{n}} L(q, x) d \mu,
$$

where $L$ is the corresponding Lagrangian of $H$. We say that $\mu \in \mathcal{I}$ is a Mather measure if

$$
\int_{\mathbb{R}^{n} \times \mathbb{T}^{n}} L(q, x) d \mu=-c_{H}
$$

Received by the editors September 29, 2009 and, in revised form, December 31, 2009.

2010 Mathematics Subject Classification. Primary 37J20, 35J70, 37J50.

The first author was partially supported by the CAMGSD/IST through the FCT Program POCTI/FEDER and by grants DENO/FCT-PT (PTDC/EEA-ACR/67020/2006 and UTAustin/MAT/0057/2008.

The second author was partially supported by Conacyt grant 83739 .

The fourth author was partially supported by NSF grants D0848378 and D0901460.

(C)2010 American Mathematical Society Reverts to public domain 28 years from publication 
A probability measure on $\mathbb{T}^{n}$ is called a projected Mather measure if it is the projection of a Mather measure. It is well known that a Mather measure is supported on a graph and is uniquely determined by the associated projected Mather measure. See Mather $[\mathrm{M}]$ for the original proof and Fathi $[\mathrm{F}$. for more background and details. The Mather set associated with $H$ is defined as

$$
\mathcal{M}=\overline{\bigcup\{\operatorname{spt}(\mu) \mid \mu \text { is a Mather measure }\}} .
$$

The Mather set is very important in the dynamical system. However, when $n \geq 3$, very little is known about its structure. Hence it is very useful to develop some approximation schemes for the Mather set so that it can be at least numerically located. So far, there have been several approximation schemes introduced by different authors (Anantharaman [A]; Evans [E1], E2]; Gomes [G1, G2]). In this note, we will focus on the one considered in [E1]. As we will discuss later, this approach might have the advantage of identifying the whole Mather set. Here is a brief description of Evans's variational scheme. For each $k \in \mathbb{N}$, assume that $v_{k} \in \mathcal{W}$ minimizes the functional

$$
\int_{\mathbb{T}^{n}} e^{k H(D v, x)} d x
$$

over the admissible class

$$
\mathcal{W}=\left\{v \in W^{1, \infty}\left(\mathbb{T}^{n}\right) \mid \int_{\mathbb{T}^{n}} v d x=0\right\} .
$$

Define

$$
\sigma_{k}=\frac{e^{k H\left(D v_{k}, x\right)}}{\int_{\mathbb{T}^{n}} e^{k H\left(D v_{k}, x\right)} d x} .
$$

It was proved in E1] that $v_{k} \in C^{\infty}\left(\mathbb{T}^{n}\right)$. Also, if $k_{j} \rightarrow+\infty$ as $j \rightarrow \infty$ and

$$
\sigma_{k_{j}} \rightarrow \sigma \quad \text { weakly as measures, }
$$

then $\sigma$ is a projected Mather measure. In addition, if

$$
v_{k_{j}} \rightarrow v \text { uniformly in } \mathbb{T}^{n},
$$

then $v \in W^{1, \infty}\left(\mathbb{T}^{n}\right)$ is a viscosity solution of the equation

$$
\sum_{1 \leq i, j \leq n} H_{p_{j}}(D v, x) H_{p_{i}}(D v, x) v_{x_{i} x_{j}}+\sum_{1 \leq i, j \leq n} H_{p_{i}}(D v, x) H_{x_{i}}(D v, x)=0 .
$$

The above equation is the so-called "Aronsson equation", which was first introduced by Aronsson in Ar1, Ar2, Ar3. It is the Euler-Lagrange equation of absolute minimizers of the functional

$$
F_{\infty}(w, U)=\operatorname{ess} \sup _{\mathbb{U}} H(D w, x) .
$$

Considering the inf-sup formula (see Contreras-Iturriaga-Paternain-Paternain [C], Gomes [G1, etc.)

$$
c_{H}=\inf _{v \in C^{1}\left(\mathbb{T}^{n}\right)} \max _{\mathbb{T}^{n}} H(D v, x),
$$

Evans's idea is quite natural. See E1] for more motivation.

Mather measures in general are not unique, so neither are the projected Mather measures. Also, equation (1.2) may have infinitely many solutions even up to a constant. Hence a natural and interesting question is the following: 
Question 1.1. How can we characterize Mather measures and solutions of equation (1.2) selected by Evans's approach?

Similar problems have been studied for other approximation schemes. See section 4 . Generally speaking, such problems are very difficult when $n \geq 2$. See Gomes G2 for some interesting computations. In this note, we will study the 1-d case. Although the low dimension situation is simple, the results we have established at least give us some hint about what might happen when $n \geq 2$.

For convenience, we will look at the Hamiltonian

$$
H(p, x)=\frac{1}{2}|P+p|^{2}+V(x)
$$

where $P \in \mathbb{R}^{n}$. It is very easy to generalize our results to all Hamiltonians satisfying assumptions (i) and (ii).

Below is the main theorem.

Theorem 1.2. Assume that $n=1$. Then $v_{k}$ will converge to a unique function $v \in C^{1}\left(\mathbb{T}^{1}\right)$ which satisfies

$$
P+v^{\prime}(x)=\operatorname{sign}(P) \sqrt{2(c(P)-V(x))^{+}} .
$$

Here $c(P) \geq \min _{\mathbb{T}^{1}} V$ is the unique number satisfying

$$
|P|=\int_{0}^{1} \sqrt{2(c(P)-V)^{+}} d x .
$$

If we further assume that $|P|<P_{0}$, where $P_{0}$ is a constant given in section 2, then $\sigma_{k}$ has the same weak limits as

$$
\tilde{\sigma}_{k}=\frac{e^{-k V}}{\int_{\mathbb{T}^{1}} e^{-k V} d x}
$$

i.e, for any $\phi \in C\left(\mathbb{T}^{1}\right)$,

$$
\lim _{k \rightarrow+\infty}\left(\int_{\mathbb{T}^{1}} \phi \sigma_{k} d x-\int_{\mathbb{T}^{1}} \phi \tilde{\sigma}_{k} d x\right)=0 .
$$

Corollary 1.3. Assume that $n=1$ and $|P|<\left|P_{0}\right|$.

(i) Suppose that $V$ has no degenerate maximum point and

$$
\Gamma=\left\{x \in \mathbb{T}^{1} \mid V(x)=\max _{\mathbb{T}^{1}} V\right\}=\left\{x_{i}\right\}_{i=1}^{d} .
$$

Then

$$
\sigma_{k} \rightarrow \sum_{i=1}^{d} \lambda_{i} \delta_{x_{i}} \quad \text { weakly in measures, }
$$

where $\lambda_{i}=\frac{\prod_{j \neq i} \sqrt{-V^{\prime \prime}\left(x_{j}\right)}}{\sum_{k=1}^{d} \prod_{j \neq k} \sqrt{-V^{\prime \prime}\left(x_{j}\right)}}$.

(ii) If $V$ has at least one degenerate maximum point and $\sigma$ is a weak limit of a subsequence of $\left\{\sigma_{k}\right\}_{k \geq 1}$, then

$$
\operatorname{spt}(\sigma) \subset\left\{x \in \Gamma \mid V^{\prime \prime}(x)=0\right\} .
$$

It is known that when $|P|>P_{0}$, the projected Mather measure is uniquely given by (2.4). Hence the case that remains open is where $|P|=P_{0}$.

Our paper is organized as follows. In section 2 , we will review some preliminary definitions, notation and results. In section 3 , we will prove our main theorem. In 
section 4, we discuss the connection with another approximation scheme and give some conjectures about the higher dimensional situation.

\section{BACKGROUND}

According to Lions-Papanicolaou-Varadhan [LP], given $P \in \mathbb{R}^{n}$, there exists a unique number $\bar{H}(P) \in \mathbb{R}$ such that the following equation has a viscosity solution:

$$
\left\{\begin{array}{l}
\frac{1}{2}|P+D v|^{2}+V(x)=\bar{H}(P) \\
v \text { is } \mathbb{Z}^{n} \text { periodic. }
\end{array}\right.
$$

The function $\bar{H}(P)$ is the so-called "effective Hamiltonian". The above existence theorem is also called the weak KAM theorem in $\mathrm{F}$ since it gives a weak formulation of the KAM (Kolmogorov-Arnold-Moser) theory. The PDE formulation of the KAM theory more or less says that if $V$ is close to a constant and $D \bar{H}(P)$ satisfies some nonresonance conditions, equation (2.1) admits smooth solutions.

When $n=1$, it is well known that $\bar{H}(P)$ is given by the following formula:

$$
\bar{H}(P)= \begin{cases}\max _{\mathbb{R}^{1}} V \quad \text { if }|P| \leq P_{0} \\ |P|=\int_{0}^{1} \sqrt{2(\bar{H}(P)-V(x))} d x \quad \text { otherwise }\end{cases}
$$

where

$$
P_{0}=\int_{0}^{1} \sqrt{2\left(\max _{\mathbb{R}^{1}} V-V(x)\right)} d x .
$$

When $|P| \geq P_{0}$, the viscosity solution of equation (2.1) is unique up to a constant. It is determined by the formula

$$
P+v^{\prime}(x)=\operatorname{sign}(P) \sqrt{2(\bar{H}(P)-V(x))} .
$$

Moreover, it is not hard to check that in this case, the viscosity solution of the Aronsson equation is also unique up to a constant and is given by the same formula.

Relations between $c(P)$ in Theorem 1.2 and $\bar{H}(P)$. It is easy to see that the following holds:

$$
\begin{cases}c(P)<\bar{H}(P) & \text { when }|P|<P_{0} \\ c(P)=\bar{H}(P) & \text { otherwise. }\end{cases}
$$

Let us denote by $\mathcal{M}_{P}$ the Mather set associated to the Hamiltonian $(\star)$ and by $\tilde{\mathcal{M}}_{P}$ its projection to $\mathbb{T}^{n}$. We hereafter write

\section{Mather measures in 1-d.}

$$
\Gamma=\left\{x \in \mathbb{T}^{n} \mid V(x)=\max _{\mathbb{T}^{n}} V\right\} .
$$

(1) If $|P| \leq P_{0}$, then $\sigma$ is a projected Mather measure if and only if it is a Borel probability measure supported on $\Gamma$. In addition,

$$
\tilde{\mathcal{M}}_{P}=\Gamma \text {. }
$$

(2) If $|P|>P_{0}, \tilde{\mathcal{M}}_{P}=\mathbb{T}^{1}$ and the projected Mather measure is uniquely given by

$$
\sigma=\frac{\lambda}{\sqrt{\bar{H}(P)-V(x)}}
$$

where $\lambda$ is such that $\int_{\mathbb{T}^{1}} \sigma d x=1$. 
The Aubry set. We also define another Euler-Lagrangian flow invariant set, which is usually larger than the Mather set but more accessible by PDE methods, namely the Aubry set.

Definition 2.1. We say that $\xi:(-\infty, \infty) \rightarrow \mathbb{T}^{n}$ is a universal characteristic if for all $t_{2}<t_{1}$ and any viscosity solution $v$ of equation (2.1), we have

$$
P \cdot\left(\tilde{\xi}\left(t_{1}\right)-\tilde{\xi}\left(t_{2}\right)\right)+v\left(\tilde{\xi}\left(t_{1}\right)\right)-v\left(\tilde{\xi}\left(t_{2}\right)\right)=\int_{t_{2}}^{t_{1}} \frac{1}{2}|\dot{\tilde{\xi}}(s)|^{2}-V(\tilde{\xi}(s))+\bar{H}(P) d s,
$$

where $\tilde{\xi}$ is the lift of $\xi$ to $\mathbb{R}^{n}$.

Definition 2.2. The Aubry set is

$$
\mathcal{A}_{P}=\bigcup\{(\dot{\xi}(s), \xi(s)) \mid s \in \mathbb{R} \text { and } \xi \text { is a universal characteristic }\} \subset \mathbb{R}^{n} \times \mathbb{T}^{n} .
$$

We denote by $\tilde{\mathcal{A}}_{P}$ the projection of the Aubry set in $\mathbb{T}^{n}$.

See $[\mathrm{F}$ for the dynamical definition and more properties of the Aubry set. Below is the projected Aubry set in 1-d.

(A1) When $|P|<P_{0}$,

$$
\tilde{\mathcal{A}}_{P}=\Gamma=\left\{x \in \mathbb{T}^{1} \mid V(x)=\max _{\mathbb{T}^{1}} V\right\} .
$$

(A2) When $|P| \geq P_{0}$,

$$
\tilde{\mathcal{A}}_{P}=\mathbb{T}^{1} .
$$

Note that when $|P|=P_{0}$, the Aubry set is larger than the Mather set.

Below is a regularity result for viscosity solutions of equation (2.1) in 1-d (Theorem $9^{\prime}$ in Ar2] $)$.

Theorem 2.3. Suppose that $n=1$ and $a<b$. If $v$ is a viscosity solution of equation (1.2) in $(a, b)$, then $v \in C^{1}((a, b))$.

\section{Proofs}

In this section, we will prove Theorem 1.2 and Corollary 1.3. Without loss of generality, we assume that $P>0$. The $P=0$ case is trivial. Since for $m \in \mathbb{N}$, $v_{m}$ is a minimizer of the functional (1.1), it satisfies the following Euler-Lagrange equation, which is integrable in 1-d:

$$
\left(e^{m\left(\frac{1}{2}\left|P+v_{m}^{\prime}\right|^{2}+V\right)}\left(P+v_{m}^{\prime}\right)\right)^{\prime}=0 .
$$

Hence there must exist a constant $h_{m} \in \mathbb{R}$ such that

$$
e^{m\left(\frac{1}{2}\left|P+v_{m}^{\prime}\right|^{2}+V\right)}\left(P+v_{m}^{\prime}\right)=h_{m} .
$$

Write

$$
u_{m}=P x+v_{m} .
$$

Lemma 3.1. Suppose that $P>0$. Then

$$
h_{m}>0 \text {. }
$$

Proof. If $h_{m} \leq 0$, then $u_{m}^{\prime} \leq 0$. So

$$
P=\int_{\mathbb{T}^{1}} u_{m}^{\prime} \leq 0 .
$$

This contradicts our assumption. 
Therefore, we may write

$$
h_{m}=e^{m c_{m}} .
$$

Lemma 3.2. Suppose that $P>0$,

$$
\lim _{k \rightarrow \infty} c_{m_{k}}=c
$$

and

$$
v_{m_{k}} \rightarrow v \quad \text { uniformly in } \mathbb{T}^{1}
$$

Then $c=c(P)$ and

$$
P+v^{\prime}(x)=\sqrt{2(c(P)-V(x))^{+}} .
$$

Proof. We assume that $u(x)=P x+v$. According to Theorem 2.3, $u \in C^{1}\left(\mathbb{T}^{1}\right)$. Owing to Lemma 3.1,

$$
u_{m}^{\prime}>0
$$

Since

$$
u_{m_{k}}^{\prime} \rightarrow u^{\prime} \quad \text { weakly in } L^{2}\left(\mathbb{T}^{1}\right)
$$

we have that $u^{\prime} \geq 0$.

Step I. For any $x$ there are $\nu_{k} \rightarrow \infty$ and $x_{k} \rightarrow x$ such that $u_{m_{\nu_{k}}}^{\prime}\left(x_{k}\right) \rightarrow u^{\prime}(x)$.

In fact, let $\nu_{k} \rightarrow \infty$ such that $\left\|u_{m_{\nu_{k}}}-u\right\|_{C\left(\mathbb{T}^{1}\right)} \leq \frac{1}{k^{2}}$.

By the mean value theorem, there is $x_{k} \in\left[x+\frac{1}{k}, x\right]$ such that

$$
\begin{aligned}
u_{m_{\nu_{k}}}^{\prime}\left(x_{k}\right) & =k\left(u_{m_{\nu_{k}}}\left(x+\frac{1}{k}\right)-u_{m_{\nu_{k}}}(x)\right) \\
& =k\left(u\left(x+\frac{1}{k}\right)-u(x)\right)+O\left(\frac{1}{k}\right) .
\end{aligned}
$$

Thus $u_{m_{\nu_{k}}}^{\prime}\left(x_{k}\right) \rightarrow u^{\prime}(x)$.

Step II. We claim that if $u^{\prime}(x) \neq 0$, then

$$
\frac{1}{2}\left|u^{\prime}\right|^{2}+V(x)=c .
$$

In fact, choose $\nu_{k}$ and $x_{k}$ as in Step I. According to Theorem 5.1 in [E1, there exists a constant $C$ independent of $m$ such that

$$
0<u_{m}^{\prime} \leq C
$$

Since $u^{\prime}(x)>0$,

$$
\lim _{k \rightarrow+\infty}\left(u_{m_{\nu_{k}}}^{\prime}\left(x_{k}\right)\right)^{\frac{1}{m_{\nu_{k}}}}=1 .
$$

From the equation

$$
e^{\frac{1}{2}\left|u_{m_{k}}^{\prime}\right|^{2}+V}\left(u_{m_{k}}^{\prime}\right)^{\frac{1}{m_{k}}}=e^{c_{m_{k}}},
$$

we derive

$$
e^{\frac{1}{2}\left|u^{\prime}\right|^{2}+V(x)}=e^{c} .
$$

Hence our claim holds. 
Step III. We claim that for any $x \in \mathbb{T}^{1}$,

$$
\frac{1}{2}\left|u^{\prime}\right|^{2}+V(x) \geq c .
$$

In fact, according to (3.2) and (3.3),

$$
\liminf _{k \rightarrow+\infty}\left(\frac{1}{2}\left|u_{m_{k}}^{\prime}\right|^{2}+V(x)\right) \geq c .
$$

Hence

$$
\liminf _{k \rightarrow+\infty} u_{m_{k}}^{\prime}(x) \geq \sqrt{2(c-V(x))^{+}} .
$$

Accordingly, (3.1) implies that

$$
u^{\prime}(x) \geq \sqrt{2(c-V(x))^{+}} .
$$

Step IV. Owing to Step II, it is clear that

$$
u^{\prime}(x) \leq \sqrt{2(c-V(x))^{+}} .
$$

Combining this with Step III, we get that

$$
u^{\prime}(x)=\sqrt{2(c-V(x))^{+}} .
$$

Hence

$$
P=\int_{0}^{1} u^{\prime} d x=\int_{0}^{1} \sqrt{2(c-V(x))^{+}} d x .
$$

Therefore,

$$
c=c(P)
$$

Lemma 3.3. Suppose that $f_{k} \in C\left(\mathbb{T}^{n}\right)$ are nonnegative. Define

$$
\mu_{k}=\frac{f_{k}}{\int_{\mathbb{T}^{n}} f_{k}} .
$$

Assume that any weak limit of $\mu_{k}$ is supported on $\Gamma$. Then for any $r>0$ and $\phi \in C\left(\mathbb{T}^{n}\right)$, the following holds:

$$
\lim _{k \rightarrow+\infty}\left(\int_{\mathbb{T}^{n}} \mu_{k} \phi d x-\int_{\mathbb{T}^{n}} \frac{f_{k}}{\int_{\Gamma_{r}} f_{k} d x} \phi d x\right)=0,
$$

where $\Gamma_{r}=\left\{x \in \mathbb{T}^{n} \mid d(x, \Gamma) \leq r\right\}$.

Proof. We write $A=\int_{\mathbb{T}^{n} \backslash \Gamma_{r}} f_{k} d x$ and $B=\int_{\Gamma_{r}} f_{k} d x$. According to our assumption,

$$
\lim _{k \rightarrow \infty} \frac{A}{B}=0
$$

Note that

$$
\left|\int_{\mathbb{T}^{n}} \mu_{k} \phi d x-\int_{\mathbb{T}^{n}} \frac{f_{k}}{\int_{\Gamma_{r}} f_{k} d x} \phi d x\right| \leq \max _{\mathbb{T}^{n}}|\phi| \frac{A}{(A+B) B} \int_{\mathbb{T}^{n}} f_{k} d x=\max _{\mathbb{T}^{n}}|\phi| \frac{A}{B} .
$$

So the lemma follows from (3.4). 
Proof of Theorem 1.2. The first part follows directly from (3.2) and Lemma 3.2. So let us prove (1.3). If $0 \leq P<P_{0}$, it is clear that

$$
c(P)<\max _{\mathbb{T}^{1}} V .
$$

Since

$$
u_{m}^{\prime}=e^{m\left(c_{m}-V(x)-\frac{1}{2}\left(u_{m}^{\prime}\right)^{2}\right)} \leq e^{m\left(c_{m}-V(x)\right)},
$$

there exist $r, \epsilon>0$ such that when $m$ is large enough,

$$
u_{m}^{\prime} \leq e^{-m \epsilon} \quad \text { in } \Gamma_{r} .
$$

Hence

$$
e^{\frac{1}{2} m\left(u_{m}^{\prime}\right)^{2}}=1+o(1) \text { in } \Gamma_{r},
$$

where $\lim _{m \rightarrow+\infty} o(1)=0$. Then (1.3) follows from Lemma 3.3.

Proof of Corollary 1.3. Corollary 1.3 follows immediately from the following easy calculation:

$$
\lim _{k \rightarrow+\infty} \frac{\int_{B_{r}} e^{-k a|x|^{2}} d x}{\int_{B_{r}} e^{-k b|x|^{2}} d x}=\frac{\sqrt{b}}{\sqrt{a}},
$$

where $r, a, b>0$.

\section{Connections with another approximation scheme}

Throughout this section, we say that $\sigma$ is a limiting measure of $\left\{\sigma_{k}\right\}$ if it is the weak limit of a subsequence of $\sigma_{k}$. Similarly, $v$ is called a limiting function of $\left\{v_{k}\right\}$ if it is the limit of a subsequence of $v_{k}$. We will discuss some connections with another approximation scheme given in Anantharaman [A, Evans E2 and Gomes G1. Below is the explicit construction in [A] and [E2]. See G1] for a related example. Let $v_{\epsilon}$ and $v_{\epsilon}^{*}$ be solutions of the two equations

$$
\left\{\begin{array}{l}
-\frac{\epsilon}{2} \Delta v_{\epsilon}+\frac{1}{2}\left|P+D v_{\epsilon}\right|^{2}+V(x)=\bar{H}_{\epsilon}(P) \\
v \text { is } \mathbb{Z}^{n} \text { periodic }
\end{array}\right.
$$

and

$$
\left\{\begin{array}{l}
\frac{\epsilon}{2} \Delta v_{\epsilon}^{*}+\frac{1}{2}\left|P+D v_{\epsilon}^{*}\right|^{2}+V(x)=\bar{H}_{\epsilon}(P) \\
v \text { is } \mathbb{Z}^{n} \text { periodic }
\end{array}\right.
$$

subject to

$$
\int_{\mathbb{T}^{n}} e^{\frac{v_{\epsilon}^{*}-v_{\epsilon}}{\epsilon}} d x=1
$$

The approximation in G1 takes a different point of view. However, it is easy to check that $\sigma_{\epsilon}=e^{\frac{v^{*}-v}{\epsilon}}$ is the same as the "stochastic invariant measure" in G1].

The following result is known (see [A], E2] and [G1]):

Theorem 4.1. If $\hat{\sigma}$ is a limiting measure of $\sigma_{\epsilon}$, then $\hat{\sigma}$ is a projected Mather measure.

If further technical assumptions are made, Anantharaman also proved a very interesting result in $[\mathrm{A}$ ] which says that $\hat{\sigma}$ minimizes a functional among all other projected Mather measures. However, those technical assumptions are usually very hard to verify. Below is a case where the assumptions in [A] can be checked. 
Example 4.2. Assume that $V$ has no degenerate maximum point. Suppose that $P=0$ or $n=1$ and $|P|<P_{0}$. Then if $\hat{\sigma}$ is a limiting measure of $\sigma_{\epsilon}$,

$$
\operatorname{spt}(\hat{\sigma}) \subset \Gamma_{M}=\left\{x \in \Gamma \mid F(x)=\min _{y \in \Gamma} F(y)\right\},
$$

where for $y \in \Gamma, F(y)=\sum_{i=1}^{n} \sqrt{-\lambda_{i}}$ and $\left\{\lambda_{i}\right\}_{i=1}^{n}$ are the eigenvalues of the Hessian $D^{2} u(y)$.

Note that the case where $P=0$ is trivial in our situation since it is easy to see that $v_{m}$ must be 0 if $P=0$.

Now let us look at the situation where $V$ has degenerate maximum points. The following result is from $\mathrm{Y} 1, \mathrm{Y} 2$.

Theorem 4.3. Assume that $P=0$ or $n=1$ and $|P|<P_{0}$. If there exists at least one $x_{0}$ such that $\Delta V\left(x_{0}\right)=0$, then

$$
\operatorname{spt}(\hat{\sigma}) \subset\left\{x \in \Gamma \mid \Delta V\left(x_{0}\right)=0\right\} .
$$

Hence the following is an easy consequence of Corollary 1.3.

Corollary 4.4. Assume that $P=0$ or $n=1$ and $|P|<P_{0}$. If there exists a unique point $x_{0} \in \Gamma$ such that $\Delta V\left(x_{0}\right)=0$, then these two approximation schemes select the same projected Mather measure $\delta_{x_{0}}$.

The really challenging project is to characterize limiting measures of $\sigma_{k}$ when $n>1$. Note that if $n=1$ and $V$ has no degenerate maximum point, then unlike the approximation scheme in [E2], A] and [G1 the variational scheme in [E1] catches the whole Mather set. This motivates us to ask the following:

Question 4.5. Is it true that under some nondegeneracy conditions, if $\hat{\sigma}$ is a limiting measure of $\sigma_{m}$, then

$$
\operatorname{spt}(\hat{\sigma})=\tilde{\mathcal{M}}_{P} ?
$$

PDE part. Let us look at possible behaviors of $v_{m}$ as $m \rightarrow+\infty$. First it is interesting to mention some results concerning the limits of $v_{\epsilon}$ in (4.1) as $\epsilon \rightarrow 0$. We assume that $v_{\epsilon}(0)=0$. According to the stability of viscosity solutions, it is easy to show that any limiting function of $v_{\epsilon}$ is a viscosity solution of the eikonal equation

$$
\frac{1}{2}|P+D v|^{2}+V=\bar{H}(P)
$$

with $v(0)=0$. This is the "vanishing viscosity method". However, the above equation could have infinitely many solutions. So an extremely interesting project is to identify solutions which are limiting functions of $v_{\epsilon}$. This is closely related to the problem of limiting measures since it is clear that

$$
\operatorname{spt}(\hat{\sigma}) \subset\left\{x \in \mathbb{T}^{n} \mid v(x)=v^{*}(x)\right\},
$$

where $v$ and $v^{*}$ are the corresponding limits of $v_{\epsilon}$ and $v_{\epsilon}^{*}$.

When $n=1$ and $|P| \geq P_{0}$, equation (4.3) has a unique solution. So there is nothing to show. When $|P|<P_{0}$, such a problem was first studied by JauslinKreiss-Moser [JKM]. A solution $v$ of equation (4.3) will be uniquely determined if we know where the phase transition (i.e., $P x+v$ changing from decreasing to increasing when $P \geq 0$ ) happens. The result in [JKM] says that phase transitions can only occur at $\Gamma_{M}$. In particular, if $\Gamma_{M}$ contains only one point, $v_{\epsilon}$ will have a unique 
limit as $\epsilon \rightarrow 0$. Bessi $[\mathrm{B}$ obtained similar results for more general Hamiltonians with $t$-dependence by different methods. Anantharaman-Iturriaga-Padilla-Sánchez AIPS extended these results to higher dimensions for when the Aubry set consists of finitely many periodic hyperbolic orbits.

Returning to our equation (1.2), unlike with the vanishing viscosity method, the connection between limiting functions and limiting measures is not very clear. When $n>1$, it may be too much to expect $v_{k}$ to have a unique limit as $k \rightarrow+\infty$. However, it is reasonable to believe that a limiting function should contain more dynamical information than an arbitrary viscosity solution of equation (1.2). The tough task is to dig out that information. Suppose that $v$ is a limiting function. From the limiting procedure, we guess that the quantity which matters should be $H(D v, x)$. Although it is an open problem as to whether any viscosity solution of equation (1.2) is indeed $C^{1}$, it was proved in $\mathrm{Y} 3$ ] that the quantity $H(D v, x)$ is well defined and upper-semicontinuous. Below is a wide conjecture in Y3.

Conjecture 4.6. Suppose that $v$ is a limiting function. Then

$$
D_{v}=\left\{x \in \mathbb{T}^{n}\left|\frac{1}{2}\right| P+\left.D v\right|^{2}+V(x)=\bar{H}(P)\right\}=\tilde{\mathcal{A}}_{P} .
$$

According to Theorem 1.2, it is easy to check that when $n=1$, the above conjecture holds. Also, it has been confirmed numerically for several 2-d examples. According to [Y3, if $w$ is a viscosity solution of equation (1.2), the set $D_{w}$ is closed and

$$
\tilde{\mathcal{A}}_{P}=\bigcap_{\{w \text { is a viscosity solution of equation (1.2) }\}} D_{w} .
$$

Hence the above conjecture actually says that Evans's variational scheme will pick out the smallest $D_{w}$.

\section{REFERENCES}

[A] N. Anantharaman, On the zero-temperature or vanishing viscosity limit for certain Markov processes arising from Lagrangian dynamics. J. Eur. Math. Soc. 6 (2004), no. 2, 207-276. MR 2055035 (2005i:82004)

[AIPS] N. Anantharaman, R. Iturriaga, P. Padilla, H. Sánchez-Morgado, Physical solutions of the Hamilton-Jacobi equation. Discrete Contin. Dyn. Syst. Ser. B 5 (2005), no. 3, 513-528. MR.2151719 (2006e:35031)

[Ar1] G. Aronsson, Minimization problems for the functional $\sup _{x} F\left(x, f(x), f^{\prime}(x)\right)$. Ark. Mat. 6 (1965), 33-53. MR0196551 (33:4738)

[Ar2] G. Aronsson, Minimization problems for the functional $\sup _{x} F\left(x, f(x), f^{\prime}(x)\right)$. II. Ark. Mat. 6 (1966), 409-431. MR0203541 (34:3391)

[Ar3] G. Aronsson, Minimization problems for the functional $\sup _{x} F\left(x, f(x), f^{\prime}(x)\right)$. III. Ark. Mat. 7 (1969), 509-512 (1969). MR0240690 (39:2035)

[B] U. Bessi, Aubry-Mather theory and Hamilton-Jacobi equations. Comm. Math. Phys. 235 (2003), no. 3, 495-511. MR1974512 (2004e:37096)

[C] G. Contreras, R. Iturriaga, G. P. Paternain, M. Paternain, Lagrangian graphs, minimizing measures and Mañé's critical values. Geom. Funct. Anal. 8 (1998), no. 5, 788-809. MR.1650090 (99f:58075)

[E1] L. C. Evans, Some new PDE methods for weak KAM theory. Calc. Var. Partial Differential Equations 17 (2003), no. 2, 159-177. MR1986317 (2004e:37097)

[E2] L. C. Evans, Towards a quantum analog of weak KAM theory. Comm. Math. Phys. 244 (2004), no. 2, 311-334. MR2031033 (2005c:81063)

[F] A. Fathi, Weak KAM theorem in Lagrangian dynamics, Cambridge University Press, in press.

[G1] D. Gomes, A stochastic analogue of Aubry-Mather theory. Nonlinearity 15 (2002), no. 3, 581-603. MR 1901094 (2003b:37096) 
[G2] D. Gomes, Generalized Mather problem and selection principles for viscosity solutions and Mather measures. Adv. Calc. Var. 1 (2008), no. 3, 291-307. MR2458239

[JKM] H. R. Jauslin, H. O. Kreiss, J. Moser, On the forced Burgers equation with periodic boundary conditions. Differential Equations: La Pietra 1996. Proc. Symp. Pure Math. 65, Amer. Math. Soc., Providence, RI, 1999. MR.1662751 (99m:35208)

[LPV] P. L. Lions, G. Papanicolaou, S. R. S. Varadhan, Homogenization of Hamilton-Jacobi equations, unpublished manuscript, circa 1988.

[M] J. N. Mather, Action minimizing invariant measures for positive definite Lagrangian systems, Math. Z. 207 (1991), no. 2, 169-207. MR.1109661 (92m:58048)

[Y1] Y. Yu, $L^{\infty}$ variational problems, Aronsson equations and weak KAM theory, Ph.D. dissertation, University of California, Berkeley, 2005.

[Y2] Y. Yu, A remark on the semi-classical measure from $-\frac{h^{2}}{2} \Delta+V$ with a degenerate potential V. Proc. Amer. Math. Soc. 135 (2007), no. 5, 1449-1454. MR2276654 (2007k:35079)

[Y3] Y. Yu, $L^{\infty}$ variational problems and weak KAM theory. Comm. Pure Appl. Math. 60 (2007), no. 8, 1111-1147. MR2330625 (2008f:49008)

Departamento de Matemática and CAmGSD, Instituto Superior Técnico, Lisboa, Portugal

E-mail address: dgomes@math.ist.utl.pt

Centro de Investigación en Matemáticas, Guanajuato, MéXico

E-mail address: renato@cimat.mx

Instituto de Matemáticas, Universidad Nacional Autónoma de México, México DF 04510, MÉXICO

E-mail address: hector@matem.unam.mx

Department of mathematics, University of California at Irvine, Irvine, California 92697

E-mail address: yyu1@math.uci.edu 